\title{
Diversidad de Agaricomycetes clavarioides en la Estación de Biología de Chamela, Jalisco, México
}

\section{Diversity of clavarioid Agaricomycetes at the Chamela Biological Station, Jalisco, Mexico}

\author{
Itzel Ramírez-López ${ }^{1}$, Margarita Villegas-Ríos ${ }^{1 \bowtie}$ y Zenón Cano-Santana ${ }^{2}$ \\ ${ }^{l}$ Laboratorios de Micología, Departamento de Biología Comparada, Facultad de Ciencias, Universidad Nacional Autónoma de México. Ciudad \\ Universitaria, Del. Coyoacán, 04510 México, D. F, México. \\ ${ }^{2}$ Grupo de Interacciones y Procesos Ecológicos, Departamento de Ecología y Recursos Naturales, Facultad de Ciencias, Universidad Nacional \\ Autónoma de México. Ciudad Universitaria, Del. Coyoacán, 04510 México, D. F., México. \\ \mvr@hp.fciencias.unam.mx
}

\begin{abstract}
Resumen. Este estudio es una contribución al conocimiento de la diversidad y estructura de los Agaricomycetes clavarioides que se desarrollan en los bosques tropicales de la Estación de Biología de Chamela, Jalisco, México. Las recolecciones se realizaron durante la temporada de lluvias de los años 2005 a 2008; se registraron datos de hábitat y morfología de los basidiomas, tipo de vegetación y sustrato donde se desarrollan, así como del patrón de crecimiento, área de distribución, abundancia y orientación e inclinación de las laderas donde se localizaron. Los 86 ejemplares registrados corresponden a 17 especies, de las cuales Physalacria changensis, P. inflata, Pterula verticillata y Scytinopogon scaber son nuevos registros para México. Scytinopogon pallescens, Pterula sp. 2 y Thelephora sp. fueron las más abundantes y 6 especies se registraron sólo 1 vez. Los datos obtenidos indican que la frecuencia con la que se hallan los basidiomas de los clavarioides en los distintos hábitats no es aleatoria, sino que su producción se da preferentemente en las laderas sur con inclinación de $21^{\circ}$ a $30^{\circ}$ y en el bosque tropical subperennifolio.
\end{abstract}

Palabras clave: macromicetos, basidiomicetos, bosque tropical.

\begin{abstract}
This study is a contribution to the biodiversity and community structure of clavarioid Agaricomycetes in the tropical forests of the Chamela Biological Station, in Jalisco, Mexico. The collections were made during the rainy seasons from 2005 to 2008 during which we recorded the morphological and ecological information, including basidiocarp morphology and patterns of growth. Likewise, records were also taken for habitat preferences, types of substrate, orientation, slope inclination, range of distribution and abundance. The 86 specimens recorded, corresponded to 17 different species, from which Physalacria changensis, P. inflata, Pterula verticillata and Scytinopogon scaber are new records for Mexico. The species S. pallescens, Pterula sp. 2 and Thelephora sp. were the most abundant, while other 6 species were observed only once. Results suggested a non-random distribution of the basidiomes, showing clear preferences of these fungi to fruit in the southern slopes of tropical evergreen forest vegetation at angles from $21^{\circ}$ to $30^{\circ}$.
\end{abstract}

Key words: macromycetes, basidiomycetes, tropical forest.

\section{Introducción}

Tradicionalmente se reconocen como hongos clavarioides los Agaricomycetes que forman basidiomas macroscópicos simples (cilíndricos o clavados) a profusamente ramificados, con coloración variable y consistencia carnosa a correosa o cartilaginosa en diferentes gradaciones. Su hábitat puede ser terrícola, humícola y lignícola, desarrollándose en bosques templados, subtropicales y tropicales (Corner, 1970; Petersen, 1988). Actualmente se reconocen poco más de 30 géneros y cerca de 800 especies, pero sólo algunos como Deflexula Corner, Pterula Fr., Lachnocladium

Recibido: 19 octubre 2011; aceptado: 27 julio 2012
Lév., Physalacria Peck, Aphelaria Corner, Scytinopogon Singer, Dimorphocystis Corner y Pterulicium Corner son predominantemente tropicales alcanzando incluso su máxima diversidad específica en estas regiones y, en otros casos, únicamente algunos de sus taxones se desarrollan en estas zonas (Corner, 1970; Petersen, 1988; Kirk et al., 2008).

En México sólo han sido registradas cerca de 20 especies de los géneros Lachnocladium, Lentaria Corner, Pterula, Ramaria Fr. ex Bonord., Macrotyphula R.H. Petersen, Scytinopogon y Thelephora Ehrh. ex Willd. para zonas tropicales de la región sureste (Pérez-Moreno y Villarreal, 1989; Villarreal y Pérez-Moreno, 1991; Guzmán et al., 1997; Guzmán, 2003; García-Sandoval et al., 2004; Pérez Trejo, 2005; González-Ávila, 2006). 
La Estación de Biología de Chamela (EBCH) se localiza en la costa del Pacífico mexicano del estado de Jalisco $\left(19^{\circ} 30^{\prime} \mathrm{N}, 105^{\circ} 03^{\prime} \mathrm{O}\right)$ que se caracteriza por tener una amplia área de vegetación de bosque tropical caducifolio (BTC) y en menor proporción de bosque tropical subperennifolio (BTSP). Las pendientes oscilan entre los 21 y $34^{\circ}$, y las altitudes no sobrepasan los $580 \mathrm{~m}$; los suelos son entiosoles jóvenes (o feozem hálpico) poco desarrollados, con poca materia orgánica y un $\mathrm{pH}$ entre 6 y 7; el clima es cálido subhúmedo $\left(\mathrm{Aw}_{0} \mathrm{i}\right)$ con una marcada estacionalidad (Bullock, 1988; Martínez-Yrízar y Sarukhán, 1993; Solís-Villalpando, 1993; García-Oliva et al., 1995, 2002; Lott y Atkinson, 2002). Los trabajos de macromicetos desarrollados en esta zona sólo comprenden registros de algunos Agaricales y Gasteromicetes (Bautista-Hernández y Aguirre-Acosta, 2004; Pulido-Pérez, 2008); antes del presente trabajo ningún Agaricomycetes clavarioide había sido registrado en la región.

\section{Materiales y métodos}

Se efectuaron 6 muestreos en la $\mathrm{EBCH}$ durante las épocas de lluvia de los años 2005 a 2008. Debido a la baja frecuencia de los basidiomas de los hongos clavarioides, los muestreos se realizaron por el método de vagabundeo recorriendo las diferentes veredas y vegetación aledaña a éstas. Una vez localizados, se registró la ubicación de los basidiomas con respecto a la vegetación, el tipo de ésta, el del sustrato (sensu Lodge, 1997 y Suárez-Duque, 2004), la orientación y pendiente de la ladera, el tipo de crecimiento (sensu Gibertoni et al., 2007), el número de basidiomas por colecta y el área cubierta por éstos.

El área cubierta por los basidiomas de un ejemplar dentro de una misma recolección $(A)$, se calculó a partir de la fórmula (Mueller-Dombois y Ellenberg, 1974):

$$
A=\pi \frac{\left(d_{1}+d_{2}\right)^{2}}{4}
$$

donde $d_{1}$ es el diámetro $1, d_{2}$ es el diámetro 2 guardando una relación de perpendicularidad.

Por otra parte, se valoró la riqueza específica y abundancia de hongos clavarioides para la EBCH y se calculó el índice de diversidad de Shannon-Wiener $\left(H^{\prime}\right)$, así como el valor de equidad de Pielou (J') (Magurran, 1991; Yanna et al., 2002; Zhou y Hyde, 2002).

Al no existir una definición consensuada de individuo para macromicetos, se tomo como referencia la ofrecida por Mungía et al. (2003, 2006), quienes lo definen como uno o más esporomas de la misma especie recolectados en una área determinada y en un mismo momento del día, si la distancia entre ellos no excede más de 60 centímetros.

Se construyó una curva de acumulación de especies para la zona, tomando como unidad de esfuerzo de muestreo una hora de colecta. Se hicieron pruebas de $\chi^{2}$ para determinar si los basidiomas se distribuían al azar respecto a las variables de vegetación, inclinación y orientación de la ladera. Para ello, se calculó la fracción de hábitats disponible de cada tipo mediante un muestreo sistemático de 335 puntos separados $10 \mathrm{~m}$ entre sí a través de la vegetación y veredas utilizadas para detectar basidiomas de los hongos clavarioides. Asimismo, se aplicó la prueba de residuos para determinar cuáles de las orientaciones y pendientes donde se desarrollaron los hongos clavarioides presentan frecuencias significativamente diferentes respecto a lo que se espera de acuerdo con la tabla de contingencia (Siegel y Castellan, 1995).

La descripción de los caracteres macromorfológicos de los ejemplares se realizó con el apoyo de los trabajos de Petersen (1988) y Delgado Fuentes et al. (2005); los colores fueron descritos con base en la guía de Methuen (Kornerup y Wanscher, 1978). Para la revisión microscópica se siguieron las técnicas propuestas por Largent et al. (1977) y Basso (2005), realizando cortes a mano con el apoyo del microscopio estereoscópico y rehidratándolos con $\mathrm{KOH}$ al 5-10\%. Las estructuras demasiado hialinas se contrastaron con floxina, azul de algodón, rojo congo o Melzer.

En la determinación de los ejemplares se utilizaron las claves y descripciones siguientes: Corner (1950, 1968, 1970), Marr y Stuntz (1973), Petersen (1975, 1981, 1988), Berthier (1985), Ping y Zhu-Liang (2003), García-Sandoval et al. (2004), Tanaka et al. (2004), (2005) y Exeter et al. (2006). El material se encuentra depositado en los herbarios FCME (Herbario Ma. Agustina Batalla, de la Facultad de Ciencias, UNAM) con algunos duplicados en MEXU (Herbario Nacional).

\section{Resultados}

Con un muestreo de 261 horas-colector durante las épocas de lluvia de los años 2005 a 2008 en la EBCH, se obtuvieron 17 taxones que corresponden a 8 géneros de Agaricomycetes clavarioides y una abundancia de 86 individuos.

Todos los taxones son registros nuevos para la EBCH y 4 lo son para México, cuya descripción se presenta en este trabajo (Cuadro 1). El resto de los taxones: Clavulina sp. 1, Clavulina sp. 2, Pterula sp. 3 y Thelephora sp. son probables nuevas especies. Las especies de Ramaria no pudieron determinarse, ya que sólo se obtuvo 1 para cada caso. Por lo que se refiere a Deflexula sp.1, aunque morfológicamente 
Cuadro 1. Morfoespecies de clavarioides presentes en la EBCH

\begin{tabular}{l}
\hline Taxones \\
\hline Clavulina sp. 1 \\
Clavulina sp. 2 \\
Deflexula sp. 1 \\
Deflexula sp. 2 \\
Lentaria surculus (Berk.) Corner \\
Physalacria changensis Rostrup* \\
P. inflata (Schwein.)Peck* \\
Pterula verticillata Corner* \\
Pterula sp. 1 \\
Pterula sp. 2 \\
Pterula sp. 3 \\
Phaeoclavulina aff. articulotela R.H. Petersen \\
R. aff. moelleriana Marr et D.E. Stuntz \\
R. aff. rainierensis (Bres. et Roum.) Corner \\
Scytinopogon pallescens (Bres.) Singer \\
S. scaber (Berk. et M.A. Curtis) D.A. Reid* \\
Thelephora sp.
\end{tabular}

corresponde a $D$. nana, presenta problemas nomenclaturales aún no definidos y en Deflexula sp. 2, Pterula sp. 1 y Pterula sp. 2 no se observó himenio a pesar de contar con ejemplares recolectados en diferentes fechas. El resto de las especies ya habían sido registradas para México.

Los valores de $H^{\prime}$ y de $J^{\prime}$ fueron de 0.976 y 0.793 , respectivamente. Las especies más comunes durante el periodo de estudio fueron Pterula sp. 2, Scytinopogon pallescens y
Thelephora $\mathrm{sp}$. , y entre las escasamente observadas estuvieron Lentaria surculus, Phaeoclavulina aff. articulotela, Ramaria aff. moelleriana y Ramaria aff. rainierensis, las cuales además formaron pocos basidiomas.

La curva de acumulación de especies muestra estabilidad después de un esfuerzo de colecta de más de 180 horas; aunque se realizaron otras exploraciones en 2009 y 2010, no se han localizaron nuevos taxones de este grupo, lo cual sugiere que el número de especies registrado de hongos clavarioides está muy cercano al que alberga la zona muestreada en la EBCH (Fig. 1).

La mayoría de las especies de este grupo de hongos desarrollan basidiomas de una altura menor a $50 \mathrm{~mm}$; no obstante, especies como Clavulina sp. 1, Pterula sp. 3 y Thelephora sp. llegan a producir basidiomas de hasta $145 \mathrm{~mm}$ de longitud. Las especies de Ramaria y Lentaria sólo produjeron de 1 a 3 basidiomas por taxón, mientras que para otras, como Physalacria inflata y Thelephora sp., se llegaron a contabilizar hasta 180 y 139 basidiomas respectivamente, en una sola recolección. Los esporomas encontrados cubrían un área que va de 0.008 a los 193 $\mathrm{m}^{2}$. El $44.2 \%$ de los ejemplares colectados presentaron un crecimiento subgregario; el $37.2 \%$ fueron gregarios y el resto presentaron un crecimiento cespitoso o solitario en igual porcentaje $(9.3 \%)$. A pesar de que en la mayoría de las especies no hay un patrón de crecimiento definido, en algunas como Physalacria inflata, $P$. changensis y las 2 de Deflexula, todos sus ejemplares se encontraron desarrollándose en forma gregaria. Además, el $84 \%$ de los ejemplares

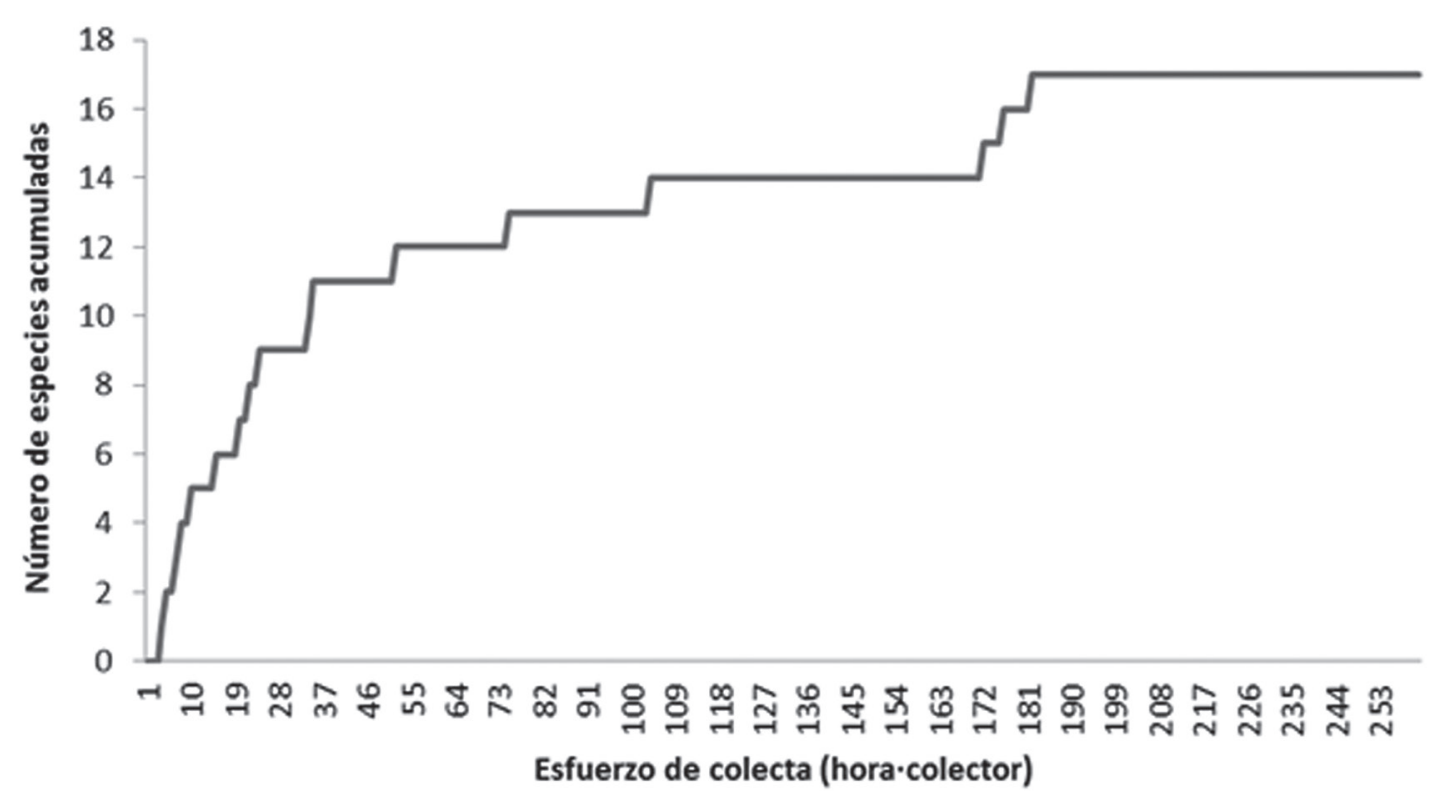

Figura 1. Curva de acumulación de especies de hongos clavarioides obtenida de 2005 a 2008. 
se encontraron desarrollándose en lugares expuestos y el $16 \%$ restante en lugares donde había la presencia de vegetación herbácea.

La distribución espacial de los basidiomas dentro de la $\mathrm{EBCH}$ no es aleatoria, en ella influyen el tipo de vegetación $\left(\chi^{2}=18.842\right.$, g.l. $=1, p<0.001$; Fig. 2$)$, la orientación $\left(\chi^{2}=20.011\right.$, g.1. $=3, p<0.001$, Fig. 3$)$ y la pendiente $\left(\chi^{2}=\right.$ 85.255 , g.l. $=2, p<0.001$, Fig. 4$)$.

Aunque los Homobasidiomycetes clavarioides se desarrollan en ambos tipos de bosque, tienen una frecuencia significativamente más alta de la esperada por azar en el BTSP, pese a que su extensión es mucho menor que la del BTC. Sin embargo, taxones como Clavulina sp. 2, Deflexula sp. 2, Pterula sp. 1, Phaeoclavulina aff. articulotela, Ramaria aff. moelleriana, Ramaria aff. rainierensis, Scytinopogon pallescens y Scytinopogon scaber sólo se registraron en el BTC, en tanto que Clavulina sp. 1, Deflexula sp. 1, Lentaria surculus y Pterula verticillata sólo se encontraron en el BTSP.

En cuanto a la frecuencia de orientación de las laderas de la $\mathrm{EBCH}$ en las que fueron registrados los basidiomas de los hongos clavarioides, un $17.8 \%$ correspondió a las laderas norte, e igual porcentaje a las oeste; un $15.6 \%$ a las este y $48.6 \%$ a las sur. Los datos estadísticos mostraron que la última fue significativamente más alta que la esperada por azar (prueba de residuos estandarizados: $[d]=3.396$, $p<0.001$; Fig. 3). Asimismo, taxones como Pterula sp. 2, Pterula sp. 3 y Thelephora sp., se registraron con mayor frecuencia en las laderas sur $(67 \%, 60 \%$ y $43 \%$ de los ejemplares, respectivamente) y Clavulina sp. 2 sólo se encontró en hábitats localizados en laderas orientadas al norte.

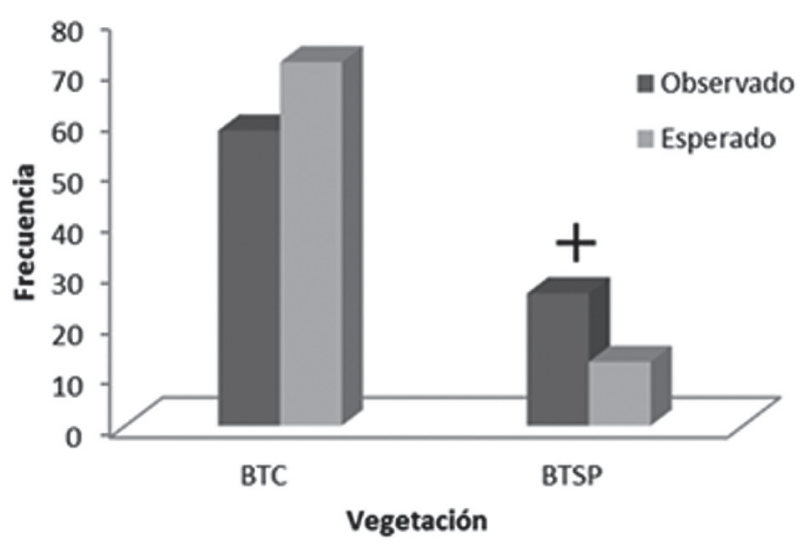

Figura 2. Frecuencia de hongos clavarioides observados y esperados en los 2 tipos de vegetación registrados en las veredas de la EBCH, Jalisco.
El 60\% de los ejemplares de clavarioides se registraron en hábitats localizados en laderas con pendientes mayores a los $20^{\circ}$, donde la frecuencia observada en laderas con una inclinación entre los 21 y $30^{\circ}$ fue significativamente más alta que la esperada ( $d=21.527, p<0.001$; Fig. 4), mientras que la de los basidiomas ubicados en laderas con pendientes entre 11 y $20^{\circ}$ fue significativamente más baja que la esperada $(d=-5.320, p<0.001)$.

Los diferentes sustratos donde se observaron desarrollándose los basidiomas de los hongos clavarioides en la $\mathrm{EBCH}$ fueron divididos en 6 categorías: restos vegetales degradados, hojas no degradadas, suelo, corteza, madera no degradada y madera degradada. Se encontró que la gran mayoría de las especies creció sobre un solo tipo de sustrato, tal como se muestra en el Cuadro 2; en cambio Physalacria changensis, Phaeoclavulina aff. articulotela y Scytinopogon pallescens se registraron sobre 2 tipos de sustratos diferentes: P. changensis sobre corteza (40\%) y madera no degradada $(60 \%), P$. aff. articulotela en restos vegetales $(50 \%)$ y madera no degradada $(50 \%)$, mientras $S$. pallescens en restos vegetales (86\%) y suelo (14\%).

\section{Descripciones de los nuevos registros para México}

Physalacria changensis Rostr., Bot. Tidsskr. 24: 206 (1902). (Figs. 5, 6)

=Physalacria tropica Corner, Ann. Bot. Mem. 1: 697 (1950).

Basidiomas pileado-estipitados, de 1-12 mm de longitud total. Parte apical de 1-9 × 1-5 mm, homogéneamente globosa cuando joven a globoso-lobulada al madurar, de color blanco a blanco amarillento (2A1-2) y en algunos

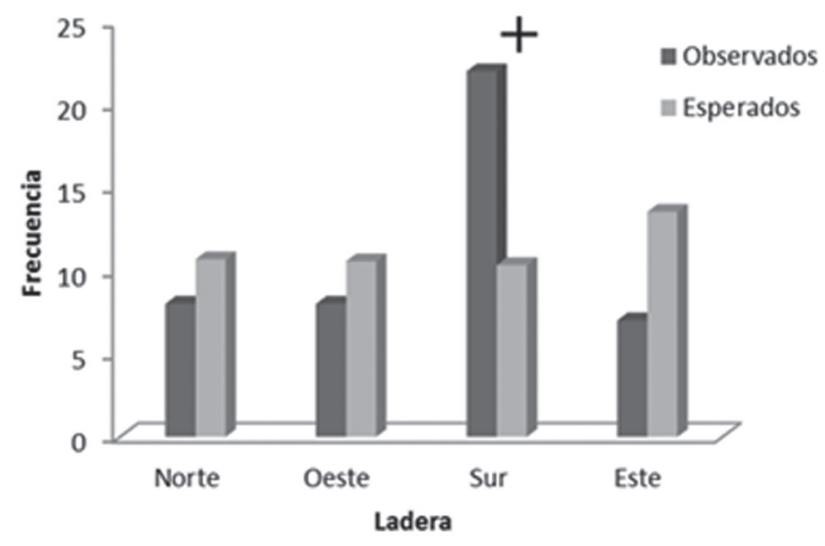

Figura 3. Frecuencia observada y esperada de hongos clavarioides en laderas con distinta orientación. El signo + denota que la frecuencia observada es significativamente mayor a la esperada (prueba de residuos estandarizados). 
Cuadro 2. Frecuencia de cada especie de hongo clavarioide presente en la $\mathrm{EBCH}$, en diferentes tipos de sustrato. Datos agrupados de 2005 a 2008

\begin{tabular}{|c|c|c|c|c|c|c|c|}
\hline & $\begin{array}{l}\text { Restos vegetales } \\
\text { degradados }\end{array}$ & $\begin{array}{c}\text { Hojas no } \\
\text { degradadas }\end{array}$ & Suelo & Corteza & $\begin{array}{l}\text { Madera no } \\
\text { degradada }\end{array}$ & $\begin{array}{c}\text { Madera } \\
\text { degradada }\end{array}$ & $N$ \\
\hline Thelephora sp. & & & 24 & & & & 24 \\
\hline Pterula sp. 2 & 14 & & & & & & 14 \\
\hline Scytinopogon pallescens & 12 & & 2 & & & & 14 \\
\hline Pterula sp. 3 & & 9 & & & & & 9 \\
\hline Physalacria changensis & & & & 2 & 3 & & 5 \\
\hline P. inflata & & & & & 3 & & 3 \\
\hline Scytinopogon scaber & & & 3 & & & & 3 \\
\hline Clavulina sp. 2 & & & 2 & & & & 2 \\
\hline Deflexula sp. 2 & & & & 2 & & & 2 \\
\hline Pterula verticilata & & & 2 & & & & 2 \\
\hline Phaeoclavulina aff. articulotela & 1 & & & & 1 & & 2 \\
\hline Clavulina sp.1 & & & 1 & & & & 1 \\
\hline Deflexula sp.1 & & & & 1 & & & 1 \\
\hline Lentaria surculus & & & & & 1 & & 1 \\
\hline Pterula sp.1 & & & & 1 & & & 1 \\
\hline Ramaria aff. moelleriana & & & & & & 1 & 1 \\
\hline$R$. aff. rainierensis & & & 1 & & & & 1 \\
\hline
\end{tabular}

casos se llega a manchar de color café amarillento, hueca, con ocasionales orificios basales en los más desarrollados; superficie externa con apariencia pruinosa. Estípite cilíndrico, de 1-8 $\times-1 \mathrm{~mm}$, concoloro a la parte apical y superficie fibrilosa haciéndose más evidente hacia la base.

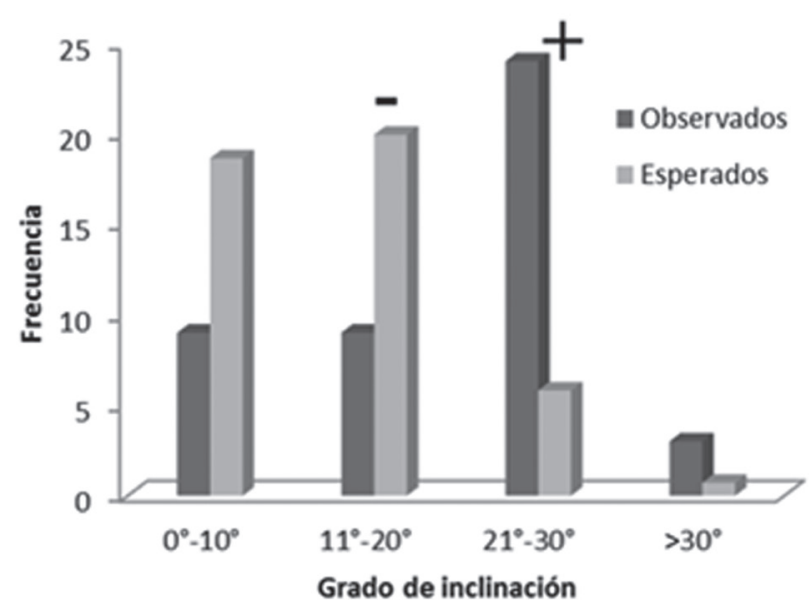

Figura 4. Frecuencia observada y esperada de los hongos clavarioides en hábitats localizados en terrenos con distinta inclinación. Los signos denotan las casillas en las que la frecuencia observada fue significativamente mayor $(+)$ o menor (-) a la frecuencia esperada (prueba de residuos estandarizados).
Consistencia membranosa hacia la parte apical y cartilaginosa-subcorreosa en el estípite. Olor ligeramente dulce.

Himenio con distribución irregular en la parte apical aunque cubriendo la mayor parte de la superficie; basidios cilíndricos a subclavados, de 16.8-29.4 × 4.2-4.9 $\mu \mathrm{m}$, tetraspóricos, hialinos, pared delgada y lisa, contenido ligeramente vacuolado y fibulados en la base; esterigmas rectos con ápices agudos, de 2.8-4.2 (-4.9) ×1.4 $\mu \mathrm{m}$, hialinos, pared lisa y delgada, y contenido homogéneo. Basidiosporas de 3.5-6.3 × 2.1-2.8 $\mu \mathrm{m}(\mathrm{E}=1.8-3.2 \mu \mathrm{m}$, $\underline{\mathrm{E}}=2.4 \mu \mathrm{m}, \underline{\mathrm{L}}=4.4 \mu \mathrm{m}, \underline{\mathrm{A}}=2.8 \mu \mathrm{m}$ ), ovaladas, hialinas, pared lisa y muy ligeramente engrosada, uni o multigutuladas, apéndice hilar apical y pequeño. Olecistidios frecuentes en la base de la parte apical e irregulares en el resto de la superficie, cilíndricos a prominentemente ventricoso-capitulados, de (35-) 37.8-56.4 (-63) × (7-) 8.4-25.2 (-27.3) $\mu \mathrm{m}$, hialinos, contenido homogéneo, pared lisa a ligeramente engrosada $(\leq 0.7 \mu \mathrm{m})$ hacia la parte capitulada, fibulados en la base. Subhimenio monomítico: hifas generativas laxamente distribuidas, de $1.4-6.3 \mu \mathrm{m}$ de ancho, pared ligeramente engrosada $(\leq 0.7$ $\mu \mathrm{m})$, contenido homogéneo, septos y fíbulas simples a intervalos muy espaciados, conexiones en $\mathrm{H}$ y ocasionalmente infladas.

Estípite conformado de un prosénquima con hifas generativas ocasionalmente infladas de (2.1-) 2.8-17.5 $\mu \mathrm{m}$ de ancho, hialinas, pared ligeramente engrosada 
(0.7-1.4 $\mu \mathrm{m})$, contenido homogéneo, septos y fíbulas simples, conexiones en $\mathrm{H}$ ocasionales y escasos septos ampuliformes. Oleocistidios frecuentes, cilíndricos a prominentemente capitulados, de 30.8-58.8 × 6.3-14 $(-21) \mu \mathrm{m}$, pared lisa y ligeramente engrosada $(0.7 \mu \mathrm{m})$, contenido homogéneo y fibulados en la base.

Hábito y hábitat. Cespitoso, lignícola, se desarrollan en bosque tropical caducifolio y bosque tropical subperennifolio a una altitud de 104 metros.

Resumen taxonómico: JALISCO, municipio de La Huerta, EBCH, vereda Búho, 9 de octubre de 2005, Villegas Ríos 2621 (FCME 26003); 1 de septiembre de 2008, Ramírez-López, I. 229 (FCME 26004); vereda Calandria, 1 de agosto de 2008, Ramírez-López, I. 177 (FCME 26005); 31 de agosto de 2008, Ramírez-López, I. 209 (FCME 26006) y 210 (FCME 26007); 18 de octubre de 2009, Ramírez-López, I. 266 (FCME 26008).

Comentarios taxonómicos. Los datos de forma, color y tamaño de los basidiomas además del tipo de basidios y tamaño de esporas de los ejemplares aquí revisados, concuerdan perfectamente con las descripciones de Physalacria changensis presentadas por Corner (1950, 1970), Berthier (1985) y Tanaka et al. (2004) no obstante, tanto en las descripciones de estos autores como en lo observado en este trabajo es evidente que la variación que presentan los cistidios en tamaño y forma es considerable. En basidiomas bien desarrollados de las recolecciones de Villegas Ríos 2621 y Ramírez-López, I. 209 y 229, únicamente se observaron cistidios subcilíndricos a clavados con la parte apical no diferenciada de (35-) 37.8-51.8 (-63) × (7-) 8.4-12.6 $\mu \mathrm{m}$; en cambio, en basidiomas con las mismas características macromorfológicas del material de Ramírez-López I. 177, sólo se observaron cistidios prominentemente capitulados, y en las recolecciones de Ramírez-López I. 210 y 266 presentaron cistidios cilíndricos hasta prominentemente capitulados de (36.6-) 42-50.4 (-70) × (11.2-) 13.3-25.2 (-29.4) $\mu \mathrm{m})$. Corner (1950) y Berthier (1985) describieron los cistidios como los presentan los primeros ejemplares (Villegas Ríos 2621, Ramírez-López, I. 209 y 229), en tanto que Tanaka et al. (2004) los han descrito con mayor amplitud, como se observaron en el segundo material (Ramírez-López I. 177).

Physalacria inflata (Schwein.) Peck, Bull. Torrey bot. Club 9: 2 (1882). (Figs. 7, 8)

ELeotia inflata Schwein., Syn. Fung. Carol. Sup.: No. 1120 (1822).

$\equiv$ Mitrula inflata (Schwein.) Fr., Elench. fung. (Greifswald) 1: 234 (1828).

=Physalacria inflata var. inflata (Schwein.) Peck, Bull. Torrey bot. Club 9: 2 (1882).
=Spathularia inflata (Schwein.) Cooke, Mycogr. t.344 (1879).

=Eoagaricus inflatus (Schwein.) L. Krieg., Bull. Maryland Acad. Sci. 3: 7 (1923).

Basidiomas pileado-estipitados, de 1-11 mm de longitud total. Parte apical globoso-lobulada, de 1-6 $\mathrm{mm}$ de diámetro, de color blanco a crema (2A1, 4A1-3), pruinosa. Estípite cilíndrico, de $1-7 \times-1 \mathrm{~mm}$, de color blanco a café rosado pálido (2A1, 4A1-2, 5B4) y superficie fibrilosa haciéndose más evidente hacia la base. Consistencia membranosa hacia la parte apical y cartilaginosa-subcorreosa en el estípite.

Himenio cubriendo la mayor parte superficie externa de la parte apical: basidios tetraspóricos, subcilíndricos a clavados, de (17.5-) 18.2-23.8 (-24.5) × 4.2-4.9 $\mu \mathrm{m}$, hialinos, pared delgada y lisa, contenido homogéneo o ligeramente vacuolado, fibulados en la base; esterigmas rectos con ápices agudos, de 2.8-4.2 × -1.4 $\mu \mathrm{m}$, hialinos, pared lisa y delgada. Basidiosporas de (2.8-) 3.5-4.2 $(-4.9) \times 2.1-2.8 \mu \mathrm{m}(\mathrm{E}=1.8-2.8 \mu \mathrm{m}, \underline{E}=2.1 \mu \mathrm{m}, \underline{\mathrm{L}}=4 \mu \mathrm{m}$, $\underline{\mathrm{A}}=2.6 \mu \mathrm{m})$, ovaladas, hialinas, pared lisa y delgada, uni o multigutuladas, apéndice hilar apical y pequeño. Oleocistidios mucronados, de 43.4-84 × (9.1-) 9.8-21 $\mu \mathrm{m}$, hialinos, pared ligeramente engrosada de $\leq 0.7 \mu \mathrm{m}$ y lisa.

Sistema hifal monomítico. Hifas generativas, de 1.44.9 (-7.7) $\mu \mathrm{m}$ de ancho, pared ligeramente engrosada $(0.7$ $\mu \mathrm{m})$, contenido homogéneo, septos y fíbulas simples a intervalos muy espaciados; conexiones en $\mathrm{H}$ ocasionales; escasos cristales pequeños de forma irregular distribuidos sobre la superficie de las hifas. Estípite conformado por un prosénquima con hifas generativas ocasionalmente infladas de (1.4-) 2.1-18.2 (-20.3) $\mu \mathrm{m}$ de ancho, hialinas, pared ligeramente engrosada $(<0.7 \mu \mathrm{m})$, contenido homogéneo, septos y fíbulas a intervalos frecuentes al igual que las conexiones en $\mathrm{H}$. Oleocistidios mucronados, de (23.1-) 26.6-56 (-70) × (7-) 8.4-14.7 (-16.8) $\mu \mathrm{m}$, pared lisa y ligeramente engrosada, contenido homogéneo.

Hábito y hábitat. Cespitoso, lignícola; se desarrolla en un bosque tropical caducifolio y bosque tropical subperennifolio a una altitud de 50 a 64 metros.

Resumen taxonómico. JALISCO, municipio La Huerta, EBCH, vereda Camino Antiguo Norte, 9 de octubre de 2005, Villegas Ríos, M. 2634 (FCME 26009); vereda Tejón, 29 de agosto de 2008, Ramírez-López, I. 185 (FCME 26010); vereda Eje Central, 1 de septiembre de 2008, Ramírez-López, I. 230 (FCME 26011).

Comentarios taxonómicos. Physalacria changensis y $P$. inflata son especies muy similares en su macromorfología, incluso comparten el mismo hábito de crecimiento, hábitat y tipo de vegetación donde se desarrollan. La diferenciación macroscópica se basa en el color café amarillento y 
los orificios basales de la parte apical del basidioma que llega a presentar Physalacria changensis; en cambio, micromorfológicamente $P$. inflata presenta esporas más pequeñas en longitud $(3.5-4.2 \times 2.1-2.8 \mu \mathrm{m})$; cistidios de la parte apical uniformemente mucronados, no ventricosos y de mayor longitud $(43.4-84 \times 9.8-21 \mu \mathrm{m})$ que $P$. changensis, tal como se muestra en la figura 9, datos que además concuerdan con las descripciones de Corner (1950) y Berthier (1985).

Pterula verticillata Corner, Ann. Bot. Mem. 1: 699 (1950). (Figs. 9, 10)

Basidiomas de 18-33 mm de longitud, muy ramificados. Ramificaciones cilíndricas a ligeramente aplanadas, de color rojo pálido (6C3), axilas en forma de $\mathrm{V}$; ápices agudos, mayoritariamente policotómicos, de color blanco amarillento (4A2). Estípite bien diferenciado, de 5-12 $\times 1-2 \mathrm{~mm}$, cilíndrico, superficie finamente fibrilosa, de color rojo pálido a café claro (7C3-6D4) y micelio de color blanco en la base. Consistencia cartilaginosacorreosa, contexto concoloro a la superficie. Olor ligero no característico.

Himenio diferenciado en las ramificaciones basales, anfígeno; basidios tetraspóricos, de 23.8-25.2 × 6.3$7.0 \mu \mathrm{m}$, clavados a subclavados, pared delgada y lisa, generalmente con contenido homogéneo o escasamente vacuolado, fibulados en la base; esterigmas rectos, de pared delgada, hialinos y contenido homogéneo. Basidiosporas de 5.6-7 × 2.8-4.2 $\mu \mathrm{m}(\mathrm{E}=2.1-3.5 \mu \mathrm{m}, \underline{\mathrm{E}}=2.6 \mu \mathrm{m}$, $\underline{\mathrm{L}}=6.3 \mu \mathrm{m}, \underline{\mathrm{A}}=3.5 \mu \mathrm{m}$ ), elipsoides a dacrioides, hialinas, pared ligeramente engrosada y lisa, contenido escasamente vacuolado y apéndice hilar lateral.

Sistema hifal en las ramificaciones dimítico. Hifas esqueléticas, de 2.1-5.6 (-8.4) $\mu \mathrm{m}$, hialinas, grosor de la pared de $0.7-1.4 \mu \mathrm{m}$, lisas, hialinas, contenido homogéneo o escasamente vacuolado. Hifas generativas, escasamente ramificadas, de 2.1-4.9 $\mu \mathrm{m}$ de ancho, hialinas, pared ligeramente engrosada $(0.7 \mu \mathrm{m})$, contenido homogéneo o escasamente vacuolado, septos espaciados con fíbulas simples; conexiones en $\mathrm{H}$ esporádicas. Cristales aplanados de forma y tamaño heterogéneos y distribuidos irregularmente en la superficie de las hifas. Estípite con hifas esqueléticas, de 2.1-3.5 (-4.2) $\mu \mathrm{m}$, hialinas, pared lisa y engrosada. Hifas generativas, de 2.1-4.9 $\mu \mathrm{m}$, hialinas, pared engrosada, contenido homogéneo, septos y fíbulas simples frecuentes y escasamente ramificados.

Hábitat y hábito. Cespitoso, terrícola; se desarrolla en un bosque tropical subperennifolio.

Resumen taxonómico: JALISCO, municipio La Huerta, $\mathrm{EBCH}$, vereda Eje Central, 9 de octubre de 2005, Villegas Ríos, M. 2633 (FCME 26012); vereda Eje Central, 31 de agosto de 2008, Ramírez-López, I. 215 (FCME 26013).
Comentarios taxonómicos. Los ejemplares determinados como Pterula verticillata, representan los primeros registros de esta especie para el país; desarrolla sus basidiomas sobre suelo dentro de un bosque tropical subperennifolio. Morfológicamente se caracterizan por la disposición verticilada de las ramificaciones del basidioma, la coloración rojo pálido o café claro decolorándose hasta blanco en las puntas, y sus esporas elipsoides de 5.6-7 x 2.8-4.2 $\mu \mathrm{m}$ que concuerdan con las descripciones de Corner $(1950,1970)$ y Petersen (1988). La única diferencia de los ejemplares estudiados respecto a las descripciones de estos autores es que aunque en la base se observa micelio, éste no forma una capa sobre el estípite.

Scytinopogon scaber (Berk. et M.A. Curtis) D.A. Reid. (1962). (Figs. 11, 12)

三 Thelephora scabra Berk. et M.A. Curtis, Amer. J. Sci. Arts, Ser. 2 11: 94 (1851).

Basidiomas ramificados de $15-40 \mathrm{~mm}$ de lontgitud. Estípite de 5-15 × 1-3 mm cilíndrico a algo aplanado, de color blanco a naranja muy claro (5A2) y base cubierta de micelio blanco con apariencia felposa. Ramificaciones aplanadas y politómicas, de color blanco a naranja muy claro o grisáceo (5A2-5B3) hacia la base; axilas redondeadas a subredondeadas; ápices agudos a subredondeados y blanquecinos. Consistencia cartilaginosa-subcorreosa y contexto concoloro a la superficie. Olor imperceptible y sabor ligeramente astringente.

Himenio anfígeno. Basidios generalmente tetraspóricos, de 20.3-28 × 4.9-5.6 $\mu \mathrm{m}$, cilíndricos a subcilíndricos, hialinos, pared delgada y lisa, base fibulada, contenido homogéneo o escasamente gutulado; esterigmas de 2.8-4.2 $\times 1.4 \mu \mathrm{m}$, hialinos, rectos, pared delgada y ápices agudos. Basidiosporas de 4.2-4.9 (-5.6) × 2.1-2.8 $\mu \mathrm{m}(\mathrm{E}=2.1-3.2$ $\mu \mathrm{m}, \mathrm{E}=2.4 \mu \mathrm{m}, \mathrm{L}=4.4 \mu \mathrm{m}, \mathrm{A}=2.6 \mu \mathrm{m})$, dacrioides, hialinas, generalmente unigutuladas al madurar, pared delgada y con quínulas menores a $0.7 \mu \mathrm{m}$, apéndice hilar lateral y tenuemente cianófilas. Subhimenio conformado por un sistema hifal monomítico, hifas generativas de $1.4-3.5 \mu \mathrm{m}$, pared ligeramente engrosada, con septos y fíbulas frecuentes, y conexiones en $\mathrm{H}$. Contexto monomítico con hifas generativas en disposición paralela, de (2.1-) 2.8-3.5 $\mu \mathrm{m}$ de ancho, pared ligeramente engrosada, contenido homogéneo, septos y fíbulas simples, conexiones en $\mathrm{H}$; presencia de escasos cristales de con forma y tamaño irregular en la superficie de las hifas. Estípite con hifas generativas, de $1.4-2.1 \mu \mathrm{m}$ de ancho, hialinas, pared ligeramente engrosada, contenido homogéneo; septos y fíbulas frecuentes, y una alta presencia de cristales sobre la superficie de las hifas en forma de placas irregulares en tamaño y forma.

Hábito y hábitat. Gregario a poco gregario, terrícola; se desarrolla en un bosque tropical caducifolio. 

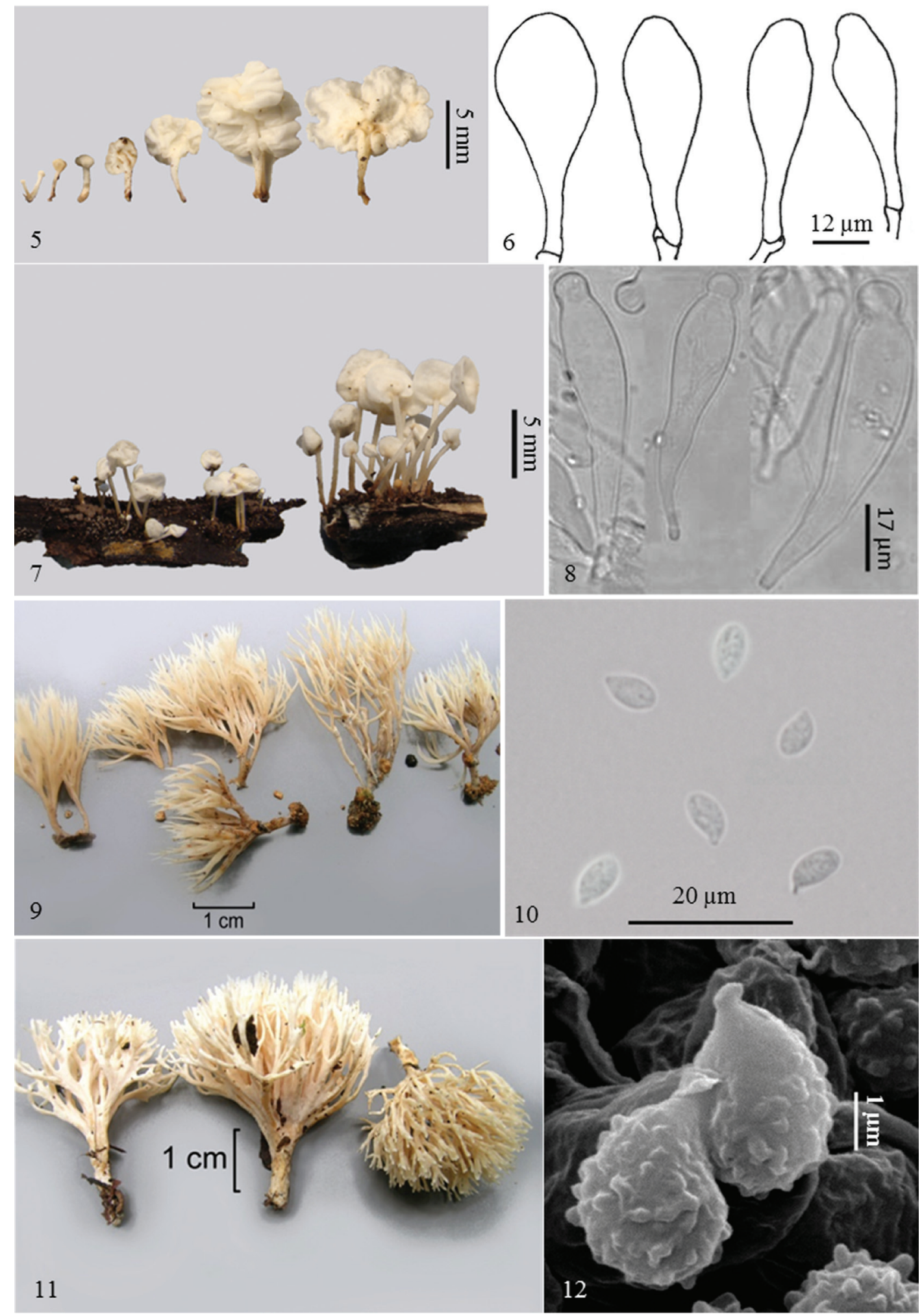

Figuras 5-12. 5-6. Physalacria changensis: 5, basidiomas (FCME 26007); 6, cistidios (FCME 26005). 7-8. Physalacria inflata (FCME 26011): 7, basidiomas; 8, cistidios. 9-10. Pterula verticillata (FCME 26012): 9, basidiomas; 10, basidiosporas. 11-12. Scytinopogon scaber (FCME 26014): 11, basidiomas; 12, basidiospora. 
Resumen taxonómico. JALISCO, municipio La Huerta, $\mathrm{EBCH}$, vereda Camino Antiguo, 9 de octubre de 2005; Villegas Ríos, M. 2630 (FCME 26014); vereda Chachalaca, 11 de agosto de 2006, Villegas Ríos, M. 2672 (FCME 26015); vereda Camino Antiguo Sur, 11 de agosto de 2006, Aguirre, Bautista y Pulido II-40 (MEXU 26345); 1 de octubre de 1977, A. Pérez J. y A. Solís M. (MEXU 11923). Comentarios taxonómicos. La macromorfología de Scytinopogon scaber es similar a $S$. pallescens en cuanto a forma, color, tamaño y consistencia de los basidiomas; no obstante, micromorfológicamente esta última especie en comparación con la primera, presenta esporas de mayor tamaño $(4.9-7 \times 2.1-2.8(-3.5) \mu \mathrm{m})$, con forma elíptica; ambas especies presentan himenio anfígeno y un sistema hifal monomítico con hifas de pared algo engrosada. Característicamente las 2 especies se desarrollan en ecosistemas tropicales donde $S$. pallescens fue previamente registrada por Sandoval-García et al. (2004) para Oaxaca, en vegetación secundaria (acahual); este es el primer registro de S. scaber para México, el cual se desarrolla en bosques tropicales caducifolios, donde se localizaron conjuntos de hasta 26 basidiomas.

\section{Discusión}

El conocimiento que se tiene en general de los Agaricomycetes con forma clavarioide en México es escaso, donde la mayoría de las especies descritas hasta el momento pertenecen a zonas templadas (Villegas y Cifuentes, 1988; Villegas, 1993; Estrada-Torres, 1994; García-Sandoval, 1997; García-Sandoval et al., 2002). Para las zonas tropicales, sólo se conocían alrededor de 20 especies (Pérez-Moreno y Villarreal, 1989; Villareal y Pérez-Moreno, 1991; Guzmán et al., 1997; Guzmán, 2003; Pérez Trejo, 2005; García-Sandoval et al., 2004; González-Ávila, 2006).

La curva de acumulación de especies indica que es apropiada la representación de la diversidad de Agaricomycetes clavarioides en la $\mathrm{EBCH}$, y que es poco el esfuerzo de muestreo que falta por realizar (Fig. 1), por lo que probablemente el número de taxones no se incremente significativamente en el futuro. Sin embargo, el que no se observen basidiomas, no significa necesariamente que la diversidad de estos hongos en el ecosistema esté concluida ya que existen especies que desarrollan esporomas entre periodos muy espaciados (Miyamoto e Igarashi, 2004; Munguia et al., 2006).

Los datos de riqueza específica obtenidos para estos hongos en la EBCH muestran que la diversidad no es tan alta como la que se observa en bosques templados o subtropicales, donde para géneros como Ramaria han sido reconocidas más de 12 especies en zonas con extensión similar (Montoya et al., 2004). En este trabajo, la mayoría de los taxones diferenciados han sido registrados únicamente para vegetación tropical y sólo algunos, como las especies de Scytinopogon, pueden también presentar una distribución subtropical. Es relevante el que 4 especies de las 17 diferenciadas, sean registros nuevos para México (Physalacria changensis, P. inflata, Pterula verticillata y Scytinopogon scaber) y otras 4, probables especies nuevas (Clavulina sp. 1, C. sp. 2, Pterula sp. 3 y Thelephora sp.).

Asimismo, destaca el hecho de que taxones como Deflexula sp. 1 y sp. 2, Pterula sp. 1 y sp. 2 no pudieron determinarse hasta especie por su muy particular biología y a pesar de haberse localizado en diferentes épocas de recolección y estados de desarrollo, no esporularon. En futuros estudios será necesario obtener nuevos ejemplares y evaluar parámetros puntuales que puedan indicar sus requerimientos en el proceso de esporulación.

Al ser éste uno de los primeros trabajos que aborda aspectos ecológicos sobre Agaricomycetes clavarioides de zonas tropicales de México en particular, los valores obtenidos para el índice de diversidad de Shannon-Wiener y de equitatividad de Pielou quedan sólo como referencia para futuras investigaciones, ya que por el momento no pueden compararse con otros trabajos similares.

Los datos ambientales que se obtuvieron en este estudio sólo consideraron aspectos macroecológicos; no obstante, fue evidente que factores como la humedad y luz están relacionados con la orientación e inclinación de la ladera y tipo de vegetación, y que a la vez tienen influencia en el desarrollo de los basidiomas de estos hongos, tal como se muestra en las figuras 2-4. Esta primera aproximación no permite aún la diferenciación puntual sobre qué factores están siendo relevantes en algunos aspectos, como el desarrollo de un himenio maduro en diferentes taxones o la preferencia por un tipo de vegetación, entre otros. Moore-Landecker (1996) y Carlile et al. (2001) señalan que ciertas cantidades de humedad, temperatura, luz y nutrimentos actúan como estímulos para la formación de las esporas y esporomas, pero si alguno o varios de éstos no se encontraran dentro del intervalo requerido para cada etapa, el desarrollo podría interrumpirse.

Respecto a la información acerca del tipo de crecimiento, número de basidiomas producidos y área que llegaron a ocupar, no se encontró una correlación entre estas variables y las especies; por tanto, es probable que se deba tanto a la distribución como a la cantidad y calidad de los recursos para la formación de los esporomas, es decir, son características propias de cada especie. A pesar de que no se encontró un patrón claro en el tipo de crecimiento, en cada recolección de Physalacria changensis, P. inflata, Deflexula sp. y Deflexula sp. 2, se observó que los esporomas crecían de manera gregaria. 
Sin embargo, Gibertoni et al. (2007) observaron que algunas especies convencionalmente agrupadas en Aphyllophorales tienden a desarrollar basidiomas agregados, lo que atribuyeron a la longitud del micelio que tenía dentro del sustrato, a la disponibilidad de recursos y a la etapa de descomposición que presenta este mismo; pero en el caso de algunos integrantes de la familia Polyporaceae encontraron esporomas agregados en sustratos sin una descomposición evidente, y en otros casos, los basidiomas tenían un desarrollo mucho más agregado en el suelo, donde la restricción espacial era mucho menor que en troncos o ramas que son unidades discretas con un tamaño determinado. Esto último, más bien lo atribuyeron a que el tipo de crecimiento puede ser una característica de las especies y en algunas ocasiones no depender de las interacciones bióticas y abióticas (Miyamoto e Igarashi, 2004; Gibertoni et al., 2007).

El presente trabajo consideró una categorización muy general de los diferentes sustratos donde se desarrollan los basidiomas de los hongos clavarioides; se observó que en el nivel de especie, pero no como grupo de hongos, hay especificidad hacia un determinado sustrato.

La literatura referente a los sustratos utilizados por los hongos es cuantiosa; la mayoría se enfoca en los degradadores de madera muerta (Senn-Irlet y Bieri, 1999; Nordén y Paltto, 2001; Heilmann-Clausen y Christensen, 2004; Küffer y Senn-Irlet, 2005; Küffer et al., 2008). Se ha tratado de establecer una clasificación de la madera con base en el estado de degradación, volumen, área y diámetro que presenta la unidad. Es interesante que en estos estudios se haya encontrado que el tamaño de la unidad de madera tiene importancia, y que son los fragmentos pequeños los que sostienen una mayor cantidad de especies de hongos, ya que su distribución en el suelo del bosque es más amplia que la de unidades grandes, como los troncos enteros de árboles caídos (Heilmann-Clausen y Christensen, 2004; Küffer et al., 2008). Sería deseable establecer una clasificación de los diferentes tipos de hábitats para los macromicetos y sobre esa base elaborar una metodología más adecuada para los muestreos con este tipo de organismos.

\section{Agradecimientos}

A la Estación de Biología de Chamela, del Instituto de Biología, UNAM, por las facilidades brindadas para la realización de este trabajo; a CONACYT, por la beca proporcionada al primer autor (210504), y al proyecto de DGAPA: PAPITT IN-203009-3, por el financiamiento otorgado. Asimismo, a Iván Castellanos-Vargas e Isael Victoria, por su asesoría en el manejo del material de campo y asesoría estadística; a los maestros en ciencias
Elvira Aguirre-Acosta, Silvia Bautista-Hernández, Sandra Castro Santiuste y Juan Andrés Pérez-Trejo, y a la bióloga Laura Izquierdo San Agustín, por su colaboración durante los muestreos.

\section{Literatura citada}

Basso, M. T. 2005. Manuale di microscopia dei Fungi. Libreria Mykoflora, Alassio. 302 p.

Bautista-Hernández, S. y E. Aguirre-Acosta. 2004. Algunas especies de lepiotáceos de la Estación de Biología de Chamela, Jalisco, México. Revista Mexicana de Micología 18:39-45.

Berthier, J. 1985. Physalacriaceae du Globe (Hyménomycétales clavarioides). Bibliotheca Mycologica 98:1-128.

Bullock, S. H. 1988. Rasgos del ambiente físico y biológico de Chamela, Jalisco, México. Folia Entomológica Mexicana $77: 5-17$.

Carlile, M. J., S. C. Watkinson y G. W. Gooday. 2001. The Fungi. Academic, San Diego, California. 588 p.

Corner, E. J. H. 1950. A monograph of Clavaria and allied genera. Dawson of Pall Mall, London. 740 p.

Corner, E. J. H. 1968. A monograph of Thelephora (Basidiomycetes). Nova Hedwigia Beihefte 27:1-110.

Corner, E. J .H. 1970. Supplement to a monograph of Clavaria and allied genera. Nova Hedwigia Beihefte 33:1-299.

Delgado-Fuentes, A., M. Villegas-Ríos y J. Cifuentes-Blanco. 2005. Glosario ilustrado de los caracteres macroscópicos en basidiomycetes con himenio laminar. Las Prensas de Ciencias, Facultad de Ciencias, UNAM, México, D. F. 84 p.

Estrada-Torres, A. 1994. La familia Gomphaceae (Aphyllophorales Fungi) en el estado de Tlaxcala. Tesis, Doctorado Escuela Nacional de Ciencias Biológicas, Instituto Politécnico Nacional. México, D. F. 186 p.

Exeter, R. L., L. Norvelly y E. Cázares. 2006. Ramaria of the Pacific Northwestern United States. United States Department of the Interior. Bureau of Land Management, Salem District, Oregon. 157 p.

García-Oliva, F., A. Camou y J. M. Maass. 2002. El clima de la región central de la costa del Pacífico mexicano. In Historia natural de Chamela, F. A. Noguera, J. H. Vega Rivera, A. N. García-Aldrete y M. Quesada-Avendaño (eds.). Instituto de Biología, Universidad, Nacional Autónoma de México, México, D. F. p. 3-10.

García-Oliva, F., J. M. Maass y L. Galicia. 1995. Rainstorm analysis and rainfall erosivity of a seasonal tropical region with a strong cyclonic influence on the Pacific coast of Mexico. Journal of Applied Meteorology 34:2491-2498.

García-Sandoval, R., J. Cifuentes y M. Villegas. 2004. First record of Scytinopogon from Mexico, with notes on its systematics. Mycotaxon 89:185-192.

García-Sandoval, R. 1997. Contribución al conocimiento del 
género Ramariopsis (Fungi, Basidiomycetes) en México. Tesis, Facultad de Ciencias, Universidad Nacional Autónoma de México, México, D. F. 57 p.

García-Sandoval, R., E. de Luna, A. Estrada-Torres y M. Villegas. 2002. A phylogeny of Ramariopsis and allied taxa. Mycotaxon 94:265-292.

Gibertoni, T. B., P. J. P. Santos y M. A. Q. Cavalcanti. 2007. Ecological aspects of Aphyllophorales in the Atlantic Rain Forest in Northeast Brazil. Fungal Diversity 25:49-67.

González-Ávila, P. A. 2006. Contribución al conocimiento del género Ramaria subgénero Echinoramaria y Ramaria en México. Tesis, Facultad de Ciencias, Universidad Nacional Autónoma de México, México, D. F. 119 p.

Guzmán, G. 2003. Los hongos del Edén, Quintana Roo, introducción a la micobiota tropical de México. CONABIO/ Instituto de Ecología, Xalapa, Veracruz. 334 p.

Guzmán, G., V. M. Bandala y L. Montoya. 1997. An overview on the tropical Fungi from Mexico. In Evaluation of fungal biological diversity in the tropics: systematics perspectives, K. K. Janardhanam, C. Rayerdran, K. Natarjan y D. L. Hawksworth (eds.). Science, Enfield, New Hampshire. p. 115-148.

Heilmann-Clausen, J. y M. Christensen. 2004. Does size matter? On the importance of various dead wood fractions for fungal diversity in Danish beech forests. Forest Ecology and Management 201:105-117.

Kirk, P. M., P. F. Cannon, D. W. Minter y J. A. Stalpers. 2008. Ainsworth \& Bisby's dictionary of the Fungi. CABI/ Cromwell, Trowbridge, Wiltshire. 632 p.

Kornerup, A. y J. H. Wanscher. 1978. Methuen handbook of colour. Methuen, London. 252 p.

Küffer, N. y B. Senn-Irlet. 2005. Diversity and ecology of woodinhabiting aphyllophoroid basidiomycetes on fallen woody debris in various forest types in Switzerland. Mycological Progress 4:77-86.

Küffer, N., F. Gillet, B. Senn-Irlet, M. Arango y D. Job. 2008. Ecological determinants of fungal diversity on dead wood in European forests. Fungal Diversity 30:83-95.

Largent, D., D. Johnoson y R. Watling. 1977. How to identify mushrooms to genus. III: Microscopic features. Mad River, Eureka, California. 148 p.

Lodge, D. J. 1997. Factors related to diversity of decomposer fungi in tropical forests. Biodiversity and Conservation 6:681-688.

Lott, E. J. y T. H. Atkinson. 2002. Biodiversidad y fitogeografía de Chamela-Cuixmala, Jalisco. In Historia natural de Chamela, F. A. Noguera, J. H. Vega Rivera, A. N. GarcíaAldrete y M. Quesada-Avendaño (eds.). Instituto de Biología, UNAM, México, D. F. p. 83-97.

Magurran, A. 1991. Ecological diversity and its measurement. Chapman \& Hall, New York. 179 p.

Marr, C. D. y D. E. Stuntz. 1973. Ramaria in western
Washington. Bibliotheca Mycologica 38:1-231.

Martínez-Yrízar, A. y J. Sarukhán. 1993. Cambios estacionales del mantillo en el suelo de un bosque tropical caducifolio y subcaducifolio en Chamela, Jalisco, México. Acta Botanica Mexicana 21:1-6.

Miyamoto, T. y T. Igarashi. 2004. Spatial distribution of Collybia pinastris sporophores in a Picea abies forest floor over a 5-year period. Mycoscience 45:24-29.

Montoya, A., A. Kong, A. Estrada-Torres, J. Cifuentes y J. Caballero. 2004. Useful wild fungi of La Malinche National Park. Mexico. Fungal Diversity 17:115-143.

Moore-Landecker, E. 1996. Fundamentals of the Fungi. Prentice Hall Upper Saddle River, New Jersey. 574 p.

Mueller-Dombois, D. y H. Ellenberg. 1974. Aims and methods of vegetation ecology. Wiley, New York. 547 p.

Munguia, P., G. Guzmán y F. Ramírez-Guillén. 2006. Seasonal community structure of macromycetes in Veracruz, Mexico. Ecography 29:57-65.

Nordén, B. y H. Paltto. 2001. Wood-decay fungi in hazel wood: species richness correlated to stand age and dead wood features. Biological Conservation 101:1-8.

Pérez-Trejo, J. A. 2005. Revisión taxonómica de algunas especies del género Lentaria Corner, en México. Tesis, Facultad de Ciencias, Universidad Nacional Autónoma de México, México, D. F. 67 p.

Pérez-Moreno, J. y L. Villarreal. 1989. First report of the clavarioid genera Lachnocladium and Pterula (Holobasidiomycetes) from the Mexican tropic. Micologia Neotropical Aplicada 2:123-130.

Petersen, R. H. 1975. Ramaria subgenus Lentoramaria with Emphasis on North American Taxa. Bibliotheca Mycologica 43:1-159.

Petersen, R. H. 1981. Ramaria subgenus Echinoramaria. Bibliotheca Mycologica 79:1-216.

Petersen, R. H. 1988. The clavarioid fungi of New Zealand. DSIR. Science Information Publishing Centre, Wellington [Nueva Zelandia]. 170 p.

Ping, Z. y Y. Zhu-Liang. 2003. Scytinopogon, a genus of clavarioid Basidiomycetes new to China. Mycosystema 22:663-665.

Pulido-Pérez, S. I. 2008. Contribución al estudio de los macromicetes de la Estación de Biología Chamela, Jalisco, México. Tesis, Facultad de Ciencias, Universidad Nacional Autónoma de México, México, D. F. 61 p.

Senn-Irlet, B. y G. Bieri. 1999. Sporocarp succession of soilinhabiting macrofungi in an autochthonous subalpine Norway spruce forest of Switzerland. Forest Ecology and Management 124:169-175.

Siegel, S. y N. J. Castellan. 1995. Estadística no paramétrica aplicada a las ciencias de la conducta. Trillas, México, D. F. 437 p.

Solís-Villalpando, E. 1993. Características fisicoquímicas de un 
suelo en un ecosistema tropical estacional. Tesis, Facultad de Ciencias, Universidad Nacional Autónoma de México, México, D. F. 91 p.

Suárez-Duque, D. 2004. Diversidad y análisis estructural de los Aphyllophorales del Bosque Protector "Mindo Lindi", Prov. de Pichincha, Ecuador. Lyona 7:83-89.

Tanaka, I., Y. Doi y T. Hongo. 2004. Two unusual species of Physalacria (Basidiomycetes, Agaricales) collected in New Zealand and Pazpua New Guinea during mycological expeditions by the National Science Museum, Tokyo. Mycoscience 45:143-146.

Villarreal, L. y J. Pérez-Moreno. 1991. The clavarioid fungi from Mexico, I. Addition of the genera Macrotyphula and
Typhula. Micologia Neotropical Aplicada 4:119-126. Villegas, M. 1993. Estudio taxonómico del género Ramaria subgénero Lentoramaria (fungi). Tesis, Facultad de Ciencias, Universidad Nacional Autónoma de México. México, D. F. 90 p.

Villegas, M. y J. Cifuentes. 1988. Revisión de algunas especies del género Ramaria subgénero Lentoramaria en México. Revista Mexicana de Micología 4:185-200.

Yanna, W. H. H. y K. D. Hyde. 2002. Fungal succesion on fronds of Phoenix hanceana in Hong Kong. Fungal Diversity 10:185-211.

Zhou, D. Q. y K. D. Hyde 2002. Fungal succession on bamboo in Hong Kong. Fungal Diversity 10:213-227. 